\title{
ESTIMACIÓN DE LA DEMANDA, APLICADA A UNA INNOVACIÓN DE TIPO MEMS MEDIANTE EL MODELO DE BASS
}

\author{
Estimate of demand, applied innovation by type MEMS through BASS model
}

Roberto Nava- Jiménez

Ingeniero. Universidad Autónoma de Querétaro, Querétaro - México, ronaji@cidesi.edu.mx

Clara Escamilla- Santana

Doctora Filosofía en Ciencia y Tecnología de Alimentos. Universidad Autónoma de Querétaro, Querétaro México, cescamis@prodigy.net.mx

\begin{abstract}
Cómo citar/ How to cite
Nava-Jiménez, R. y Escamilla- Santana, C. (2016). Estimación de la demanda, aplicada a una innovación de tipo MEMS mediante el modelo de Bass. Revista CEA, 2(3), 49-59.
\end{abstract}

Recibido: 30 de septiembre de 2015

Aceptado: 12 de noviembre de 2015

\section{Resumen}

El avance en paralelo que han tenido la mecánica y la electrónica ha creado una nueva tecnología, conocida como de micro maquinado; lo que ha permitido hacer diseño y fabricación de estructuras eléctricas y mecánicas bajo el nombre de Sistemas microelectromecánicos. Esta tecnología de orden disruptivo tiene un potencial sobresaliente para ser empleada en los dispositivos electrónicos, que se incorporan en alguna parte de nuestro cuerpo. ¿Cómo determinar el grado de adopción de los MEMS, siendo esta la tecnología de tipo habilitadora (enabling technology) aplicada a un producto en el campo de la tecnología que se lleva puesta (wearable technology)? Este trabajo propone el uso del modelo de Bass como herramienta para definir el grado de difusión, con el cual poder identificar la tendencia de adopción del reloj inteligente (smart watch) en distintos países del mundo y con ello una aproximación del tamaño de mercado, haciendo prospectiva en el rubro de los productos de consumo como uno de los sectores más promisorios en demanda futura, también es mostrado el efecto innovación versus imitación, siendo estos factores relevantes para la elaboración de estrategias de marketing y pronósticos de demanda en un nuevo producto.

Palabras clave: Modelo de Bass, tecnología disruptiva, tecnología habilitadora, MEMS, prospectiva.

\begin{abstract}
The advance in parallel that mechanics and electronics have gone through has created a new disruptive technology, known as micro machining; it has enhanced the design and manufacture of electrical and mechanical structures known under the name of Microelectromechanical Systems: MEMS. This disruptive technology has a significant potential to be applied on wearable electronics. How to determine the potential market of MEMS as an enabling technology applied to a product of wearable electronics? This paper proposes the use of the Bass model as a tool to define the degree of diffusion to identify the trend of adoption of the smart watch in different countries of the world and therefore the approach of market size, as a prospective study applied to consumer products, being these as the most promising use with future demand. It also shows the innovation vs. imitation effect,
\end{abstract}


being these factors relevant to the development of marketing strategies and forecast demand of a new product.

Keywords: Bass model, disruptive technology, enabling technology, MEMS, forecasting.

\section{INTRODUCCIÓN}

Para Ehrfeld W. y Ehrfeld U. (2001), el término 'dinámica' o 'fuerza de cambio', conocido hoy en día como mega tendencia, es aquel concepto que agrupa las grandes transformaciones en el sentir de las gentes y sus interpretaciones sobre el mundo en que habitan, propiciadas por las nuevas tecnologías y avances tecnológicos en general. Una de estas grandes megas tendencias tecnológicas son los Sistemas Microlectromecánicos (Microelectromechanical Systems: MEMS), máquinas inteligentes a nivel micrométrico que combinan sensores $y$ actuadores, estructuras mecánicas y electrónicas para recoger información del medio y responder a ello, por lo que los MEMS como tecnología de orden disruptivo está ofreciendo la oportunidad de generar nuevas industrias y progreso en la sociedad afectando sus valores, actividades y preferencias (Baltes, Brand, Fedder, Hierold, Korvink y Tabata, 2004). Para Kondratieff (1984), el orden académico debe resaltar la importancia del análisis en cuanto al potencial de mercado que genera el desarrollo de tecnologías de orden disruptivo, así también el desafío que representa la prospectiva para la difusión de las innovaciones a nivel de adopción por el mercado (Bower y Christensen, 1995).

Desde el automóvil hasta la lavadora y el celular, las nuevas tecnologías requieren de tiempo para madurar y crecer en la generación de las ideas, teorías, definición de conceptos, métodos de prueba, diversos prototipos de prueba de orden alfa, beta y gama, muchas de ellas, quizás la mayoría, fracasan en este ciclo evolutivo. Considérese como ejemplos a los localizadores y casetes de ocho pistas, estos desarrollos fungieron de guía o base como tecnología intermedia permaneciendo limitadas en cuanto a ejecución más no en cuanto al concepto. La gente deseaba comunicación móvil y música portable, a esta tecnología de los celulares y al walkman le tomó cierto tiempo de maduración para llegar al concepto ideal requerido; este proceso se ve acelerado de forma recurrente cuando una tecnología irrumpe comercialmente, existiendo adopción por la sociedad con procesos exitosos de aumento en la utilidad y en respuesta a una disminución en el costo de producción, gracias a la capacidad de manufactura, por ende también la línea de adopción por parte de las distintas clases de consumidores en el tiempo se ve rápidamente insertada, esto da pie a que explote el consumo por parte del mercado de manera cada vez más pronta. Para Naisbitt y Aburdene (1990) esta manifestación se define como "aquellas grandes fuerzas en el desarrollo humano y tecnológico que afectarán el futuro en todas las áreas de la actividad humana, en un horizonte de diez a quince años».

Bonato, Mork, Sherril y Westgaard (2003) resaltan la tendencia futura en elementos cada vez más diminutos para la detección de variables físicas, así como una evolución en la transmisión de datos vía inalámbrica, empleando en gran medida como tecnología habilitadora (enabling technology) a los MEMS, lo cual ha hecho posible el desarrollo y la creación de una nueva generación de sistemas para monitorización de las condiciones de orden fisiológico del ser humano acorde a su actividad diaria, ya sea normalmente en casa o en ambientes exteriores conocida hoy día como tecnología que se lleva puesta (wearable technology). La tendencia más presente en el mercado de productos desarrollados bajo esta tecnología al alcance del consumidor ordinario está empezando a formar parte de la lista diaria de artículos personales, ejemplo de ello, son los relojes de tipo inteligente (smart watch), los cuales poco a poco están haciendo su entrada en la sociedad, en las tiendas y en los deseos de todos aquellos usuarios de teléfonos 
inteligentes (smart phones), los cuales se encuentran presentes en todos los estratos sociales.

Por lo anterior, la pregunta de investigación que guía este estudio es la siguiente: ¿Cómo determinar el grado de adopción de los MEMS, siendo esta la tecnología de tipo habilitadora aplicada a un producto en el campo de la tecnología que se lleva puesta? Actualmente la información sobre el potencial de mercado de cualquier tecnología de orden disruptivo que surja es importante, ya que las empresas requieren invertir en tecnologías con mercados prometedores y rentables en la inversión en I\&D se recupere rápidamente.

El objetivo de este artículo se orienta al análisis del grado de difusión en cuanto a la penetración y explotación comercial de los MEMS hacia su inclusión en el reloj inteligente, empleando el modelo de Bass como herramienta que permita identificar el patrón de adopción del producto en un horizonte de tiempo dado.

\section{REVISIÓN TEÓRICA}

Los MEMS, también conocidos como microsistemas, son definidos típicamente como dispositivos microscópicos diseñados, fabricados y utilizados para interactuar o producir cambios dentro de un ambiente controlado (Fujita, 1996). Estos pequeños y más sofisticados dispositivos que piensan, actúan, miden y se comunican están reemplazando a los dispositivos actuales tradicionales en muchas aplicaciones. ¿Cuál es la motivación para usar microsistemas? El uso de microsistemas ofrece un menor consumo de potencia, alto desempeño, peso reducido y un costo más bajo que los dispositivos utilizados actualmente. La técnica de fabricación Ilamada batch (Malshe, O’Neal, Singh, Brown, Eaton y Miller, 1999), baja los costos de ensamble y manufactura, reduce el tamaño y su peso, lo que resulta en menos consumo de potencia e incrementa la flexibilidad del diseño de sistemas. El reducir el tamaño y peso de un dispositivo permite que puedan usarse múltiples componentes en serie o paralelo para aumentar la funcionalidad, capacidad y fiabilidad del dispositivo.

Para (Fujita, 1998), los microsistemas se pueden categorizar en seis distintos tipos: sensores, dispositivos MEMS diseñados para medir cambios e interactuar con el ambiente, estos microsistemas incluyen sensores químicos, térmicos, ópticos y de movimiento o tipo inercial. Actuadores, son un grupo de dispositivos diseñados para proporcionar un estímulo a otros componentes o dispositivos MEMS, en los microsistemas los actuadores son operados electrostáticamente o térmicamente. MEMS RF, son una clase de dispositivos usados para transmitir alta frecuencia, es decir, señales de radio frecuencia; los dispositivos también incluyen interruptores, capacitores, antenas, etc. MEMS ópticos, son dispositivos diseñados para dirigir, reflejar, filtrar y/o amplificar la luz, estos componentes incluyen interruptores ópticos y reflectores. MEMS para microfluidos, son dispositivos diseñados para interactuar con fluidos, dispositivos como bombas y válvulas, se han diseñado para trabajar con pequeños volúmenes de fluido. BioMEMS, son dispositivos que, como muchos de los MEMS para microfluidos, son diseñados para interactuar con muestras biológicas, dispositivos como estos son diseñados para interactuar con proteínas, células biológicas, reactivos médicos, etc. y pueden usarse para suministrar medicamentes u otro análisis médico en sitio.

Las anteriores seis áreas representan aplicaciones totalmente diferentes de dispositivos MEMS que actualmente se encuentran en uso o en desarrollo para aplicaciones en diferentes tipos de industrias y mercados, tales como la automotriz, medica, industrial y de consumo, siendo una tecnología de tipo habilitadora para aquellas innovaciones que estos productos representen el ofrecer cambiar los hábitos de la gente, ya que detrás de estos productos existe una tecnología probada gracias a la infraestructura de desarrollo, calidad y confiabilidad de los 
diferentes sistemas que se encuentran integrados en ésta como plataforma multipropósito (Baltes et al., 2004).

Para la agencia IHS Newsroom (2014), la tecnología que se lleva puesta está emergiendo de manera tan acelerada que ya ha sido considerada como la siguiente gran tendencia para los MEMS hacia su uso en los productos para el mercado de consumo después de los teléfonos inteligentes y las tablas. Su éxito comercial estará enmarcado por el tamaño de mercado que abarque, así como ser viable económicamente es decir que este soportada por los MEMS como tecnología habilitadora.
La utilización de los MEMS en: 1) relojes inteligentes, 2) monitores cardiacos y de ejercicio, 3) gafas inteligentes (smart glasses) y 4) cámaras de acción y ropa inteligente (smart clothing), destacan como aquellas aplicaciones finales donde estas complementan como una extensión natural de los teléfonos inteligentes, siendo la más promisoria en tamaño potencial de demanda en MEMS, la primera de ellas (ver Figura 1). En un futuro se estima que nuevas generaciones de sensores tipo MEMS incursionen en el monitoreo del medio ambiente, lo cual mejorará la experiencia de la actividad al aire libre en el ser humano (MEMS \& Sensors for Wearables Report, 2014).

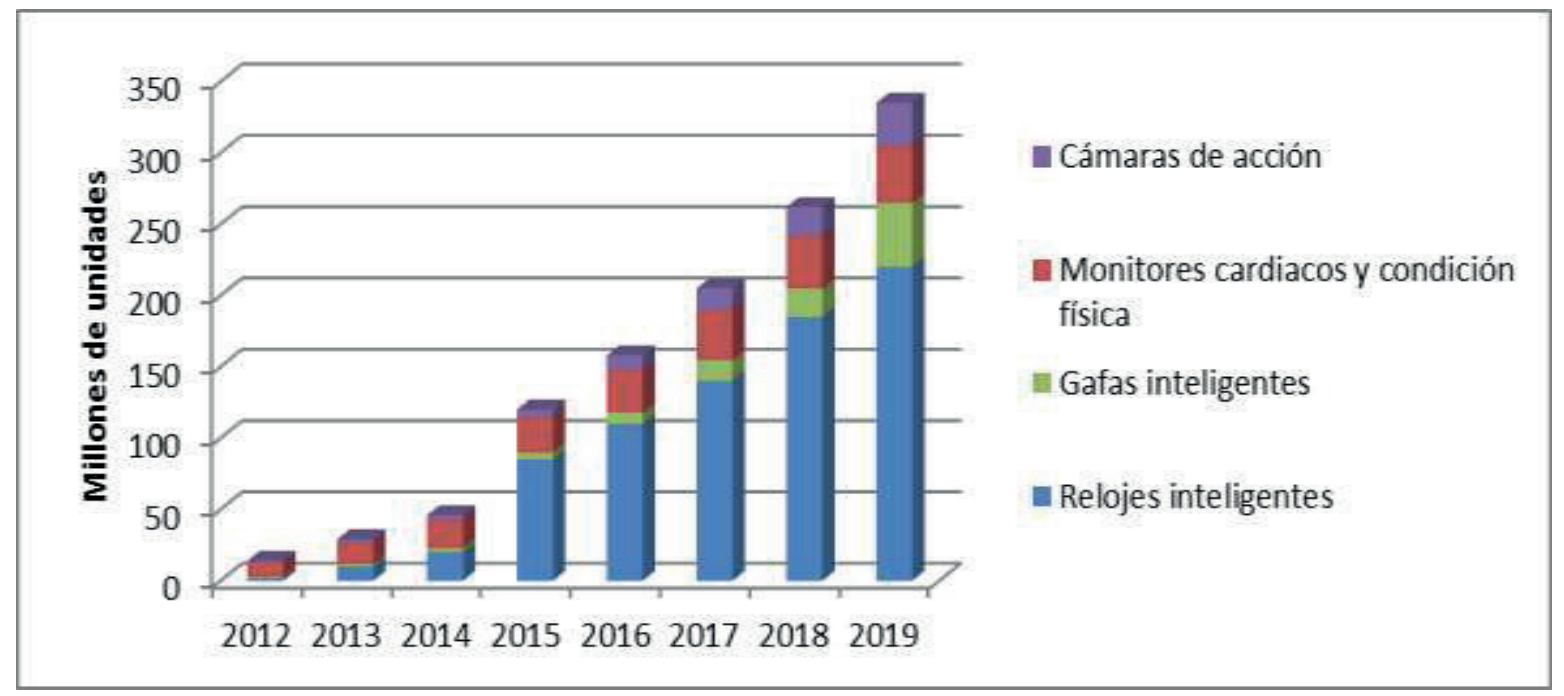

Figura 1. MEMS en productos electrónicos que se llevan puestos

Figure 1. Electronic products MEMS Wearable

Fuente: MEMS Journal, Agencia de datos IHS (2014).

Para Mahajan y Muller (1979), la estimación de la demanda futura de un producto existente en el mercado es una tarea compleja, pero no caben dudas de que es aún más compleja la tarea de predecir la demanda potencial de un producto nuevo, desconocido hasta el momento en el mercado, como lo es la situación presente para los relojes inteligentes mediante su introducción al consumidor potencial por empresas como Samsung Sony, Apple, Google, Nissan, Qualcomm (ver Figura 2). 


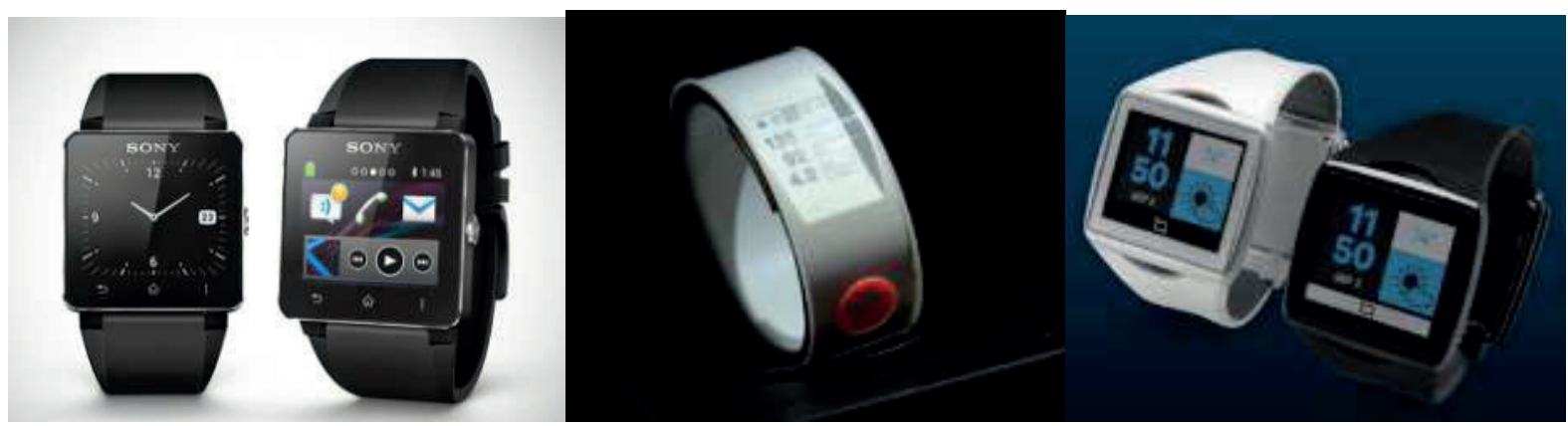

Figura 2. Reloj inteligente de Sony, Nissan y Qualcomm, respectivamente

Figure 2. Smart watch Sony, Nissan and Qualcomm, respectively.

Fuente: Mundocontact.com (2016).

A finales de la década de los años 60, Frank Bass desarrolló un modelo matemático conocido como el modelo de difusión de Bass, que ha sido la piedra angular de las distintas técnicas de estimación de la demanda de nuevos productos a lo largo de los últimos 40 años. Su utilización, desde su nacimiento, ha tenido una inmensa influencia sobre la prospectiva y el management, particularmente en los últimos 10 años, dado el notable acortamiento del ciclo de vida de los productos como consecuencia del incesante y creciente flujo de innovación tecnológica (Ofek, 2005).

Para Rogers (2003), Bass se inspiró en la teoría de la difusión de la innovación que clasifica a los individuos en cinco categorías de acuerdo con el momento en el que adoptan un nuevo producto: 1) innovador; 2) adoptante temprano; 3) mayoría temprana; 4) mayoría tardía; y 5) rezagado. A partir de esta clasificación, que segmenta a los consumidores en función del momento del ciclo de vida del producto en el que deciden comprar el mismo, Bass formalizó matemáticamente su modelo para luego probarlo con datos de once productos de consumo durable obteniendo resultados significativos. Por lo que la interpretación del modelo de Bass desde el aspecto metodológico permite estimar el número de consumidores que adoptarán (comenzarán a comprar) un nuevo producto a lo largo del tiempo, resaltando esta consideración más aún, especialmente con nuevos productos con pocos antecedentes en datos de ventas (Bass, 2004). En su trabajo titulado A New Product Growth Model for Cosumer Durables demostró que su aplicación se extiende a toda clase de productos y servicios, a B2B (Business to Business) y así como también a productos y nuevas tecnologías, tales como chips, productos médicos o maíz híbrido, etc. (Radojicic y Markovic, 2009).

\section{MODELO MATEMÁTICO DE BASS}

De acuerdo con el modelo de Bass, el número de consumidores que compran un nuevo producto en un momento determinado del tiempo es función de la demanda de los individuos innovadores, es decir, los que adquieren el nuevo producto independientemente de lo que hagan otros consumidores, y de la demanda de los imitadores o los que consumen un producto porque los demás lo hacen.

Matemáticamente, el modelo de Bass se define de la siguiente manera:

$S(t)=[p+(q / m) N(t-1)][m-N(t-1)]$

Donde:

$N(\mathrm{t})=$ número de consumidores que adoptaron el producto en el momento $t$.

$\mathrm{S}(\mathrm{t})=$ número de nuevos consumidores que adoptaron el producto en el periodo t. 
Consecuentemente:

$S(t)=N(t)-N(t-1)$

Los parámetros del modelo son:

m: máximo número de consumidores que pueden adquirir el producto (lo que no significa toda la población del país o región bajo análisis, sino solo los potenciales compradores).

p: coeficiente de innovación. Es la probabilidad que un innovador compre o adopte el producto en el periodo «t» (puede interpretarse como la tasa a la que un consumidor compra espontáneamente el producto).

q: coeficiente de imitación. Es la probabilidad que un imitador adopte el nuevo producto. Este coeficiente captura el efecto boca en boca, es decir, la comunicación que hay entre los innovadores y los imitadores, por lo cual estos últimos aprenden y copian a los primeros (también llamado efecto contagio). Este coeficiente refleja el efecto que pueden producir los consumidores ya existentes sobre los potenciales nuevos consumidores, por lo cual también recibe el nombre de coeficiente de influencia interna, a diferencia de «p» que recibe el nombre de coeficiente de influencia externa.

De la ecuación (1) se puede interpretar que $S(t)$, el número de nuevos consumidores que adquieren el producto en el momento t, es igual al producto entre la probabilidad que un nuevo consumidor adquiera el producto en el momento $\mathrm{t}$ (primer corchete) y el número de consumidores que todavía no han adoptado el producto (segundo corchete). Es importante destacar que, en este primer corchete, «q» (la probabilidad que un imitador adopte el producto) esta multiplicado por la proporción de consumidores que ya han adquirido el producto, con lo cual se refleja la influencia de los mismos en los nuevos consumidores o imitadores.

Dados los parámetros «m», «p» y «q» es posible graficar la curva de adopción de un nuevo producto, generalmente llamadas curvas «S» (por su forma sigmoidal). Intuitivamente, un valor elevado de «p» indica que el nuevo producto será adoptado rápidamente, aunque tenga una baja probabilidad de imitación (ver curva 1 de la Figura 3). Mientras que un bajo nivel de $p$ hará que la adopción sea más lenta, aunque «q» tenga un valor elevado, debido a que los imitadores tienen un reducido grupo de innovadores a quien copiar (ver curva 2 de la Figura 3). Del gráfico también se observa que en el caso de la curva 1, se alcanza el pico de consumidores rápidamente aproximadamente en el periodo cuatro, mientras que en la curva 2 , el pico recién llega aproximadamente para el periodo veinticinco.

En la formulación del modelo es interesante destacar que el número de imitadores crece primero a tasa creciente y luego a tasa decreciente, hasta llegar a un pico de ventas (ver Figura 4). A diferencia de otros modelos de predicción de demanda, la aplicación del modelo de Bass no requiere información histórica de ventas de los productos, ya que los coeficientes del modelo pueden ser determinados en referencia a otra tendencia tecnológica que tenga aspectos similares a la que se desee prospectar, por lo cual es posible su utilización como herramienta para el pronóstico de productos aún no existentes en el mercado o que aún no cuentan con información de ventas, debido a su poco tiempo de introducción al mercado, tal como se explorará su uso hacia la demanda de MEMS sobre el reloj inteligente. 


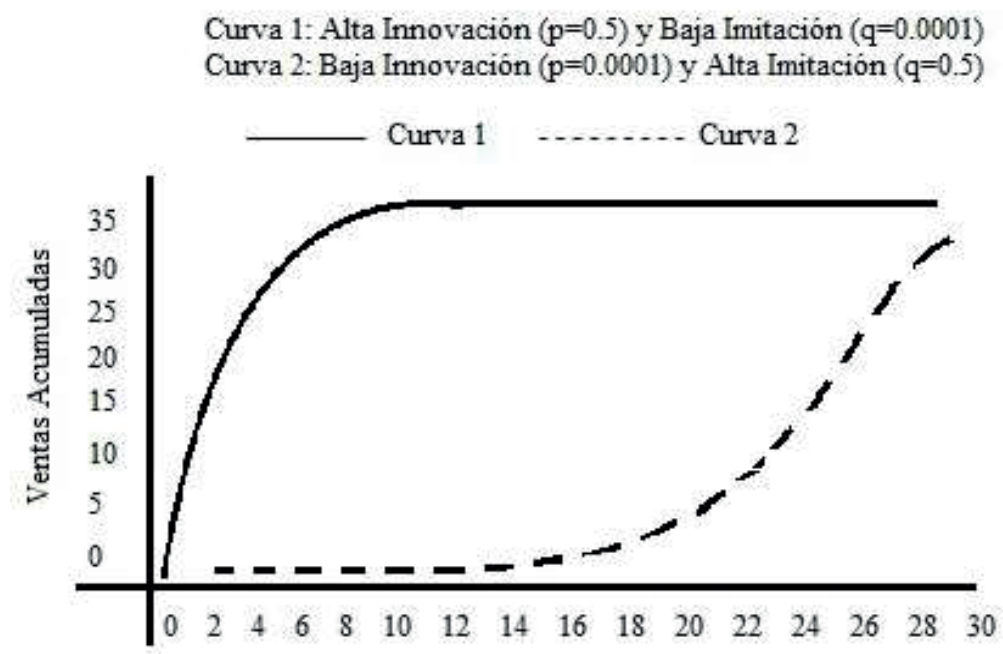

Figura 3. Patrones de adopción aplicando el modelo de Bass

Figure 3. Adoption patterns applying Bass model

Fuente: Weissmann (2008).

\section{METOdOLOGÍA}

Para focalizar la difusión esperada de la tecnología de los relojes inteligentes, y a partir de ahí estimar la demanda sobre la tecnología tipo MEMS, se ejecutaron diversas corridas en Excel para conocer el patrón de adopción S(t) en cuatro países, bajo su propia función de consumidores en adoptar el producto en el tiempo, obteniendo diferentes tendencias de difusión para los mercados potenciales de los países de México, Brasil, España y E.E.U.U. La estimación de los parámetros de innovación e imitación se obtienen en referencia a lo que fue el grado de difusión que alcanzó un producto similar, el reloj digital de funciones (ver Tabla 1).

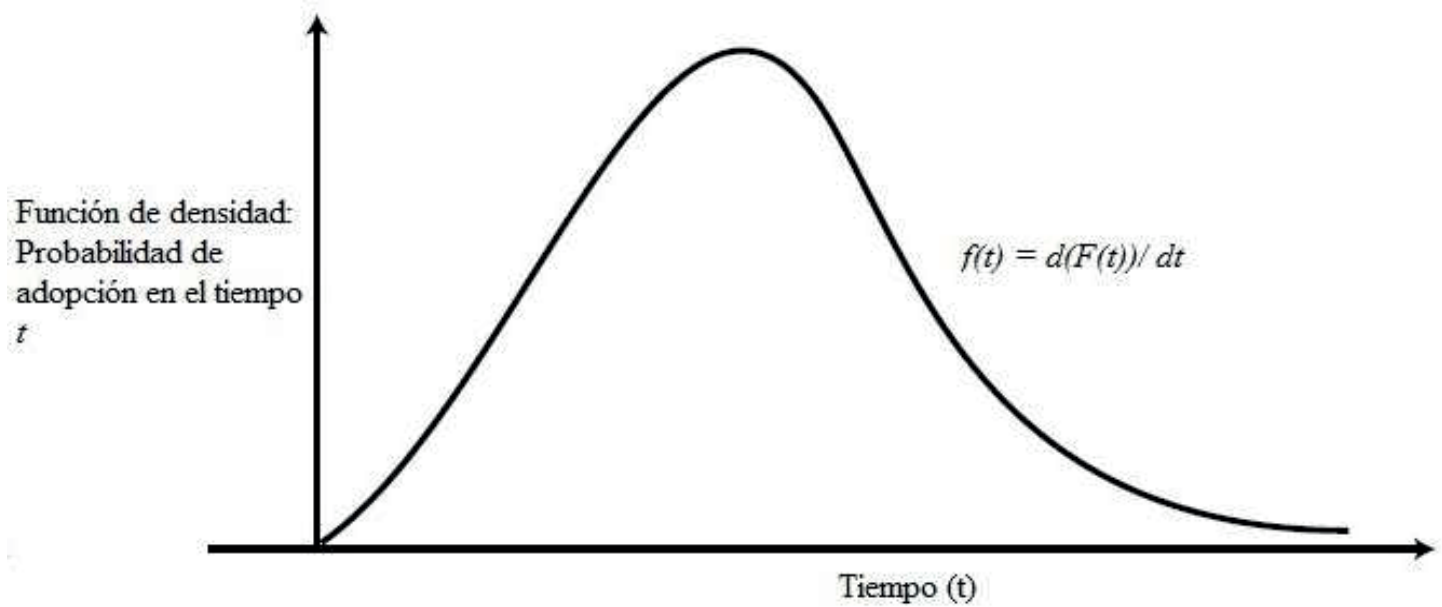

Figura 4. Tendencia de que el mercado adopte el producto en el tiempo preciso $t$

Figure 4. The market trend to adopt the product in t time

Fuente: Rogers (2003). 
Tabla 1. Parámetros para uso del modelo de Bass

Table 1. Parameters for use Bass model

\begin{tabular}{|c|c|c|c|c|}
\hline País & $\begin{array}{c}\text { Máximo número } \\
\text { potencial de } \\
\text { consumidores }(\mathrm{m})\end{array}$ & $\begin{array}{c}\text { Coeficiente de } \\
\text { innovación }(\mathrm{p})\end{array}$ & $\begin{array}{c}\text { Coeficiente de } \\
\text { imitación }(\mathrm{q})\end{array}$ & $\mathrm{S}(\mathrm{t})=[\mathrm{p}+(\mathrm{q} / \mathrm{m}) \mathrm{N}(\mathrm{t}-1)][\mathrm{m}-\mathrm{N}(\mathrm{t}-1)]$ \\
\hline México & $60,000,000$ & 0.006 & 0.8 & $\mathrm{~S}_{1}(\mathrm{t})$ \\
\hline Brasil & $100,000,000$ & 0.01 & 0.8 & $\mathrm{~S}_{2}(\mathrm{t})$ \\
\hline España & $40,000,000$ & 0.02 & 0.7 & $\mathrm{~S}_{3}(\mathrm{t})$ \\
\hline EE. UU. & $300,000,000$ & 0.027 & 0.6 & $\mathrm{~S}_{4}(\mathrm{t})$ \\
\hline
\end{tabular}

Fuente: International Telecomm Union Report (2001-2007) y www.populationpyramid.net

\section{RESULTADOS Y DISCUSIÓN}

Las proyecciones calculadas en Excel bajo el modelo de Bass muestran una tendencia para México y Brasil (ver Figuras 5 y 6 ) al arranque de la curva « $\mathrm{S}_{1}(\mathrm{t})$ y $\mathrm{S}_{2}(\mathrm{t})$ » acorde a un factor de innovación reducido, por lo que la curva de adopción de la masa crítica arranca de forma no tan acelerada, tan solo 20\% para México y $30 \%$ para Brasil sobre el mercado potencial de consumidores en los primeros cuatro años respectivamente, lo que representa una masa crítica potencial de consumidores de tipo innovador de 12 millones y 30 millones respectivamente.

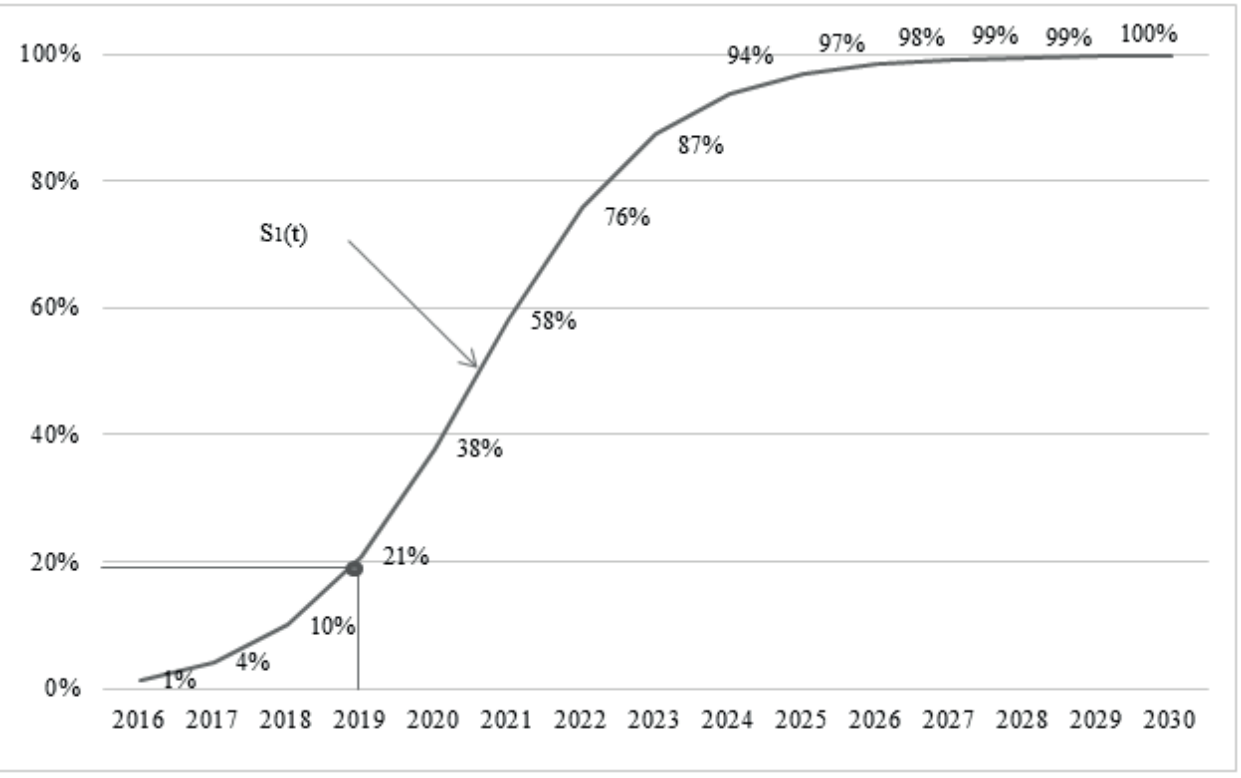

Figura 5. Curva de difusión esperada para la adopción del reloj inteligente en México

Figure 5. Expected diffusion curve for the adoption of smart watch in Mexico

Fuente: elaboración propia. 


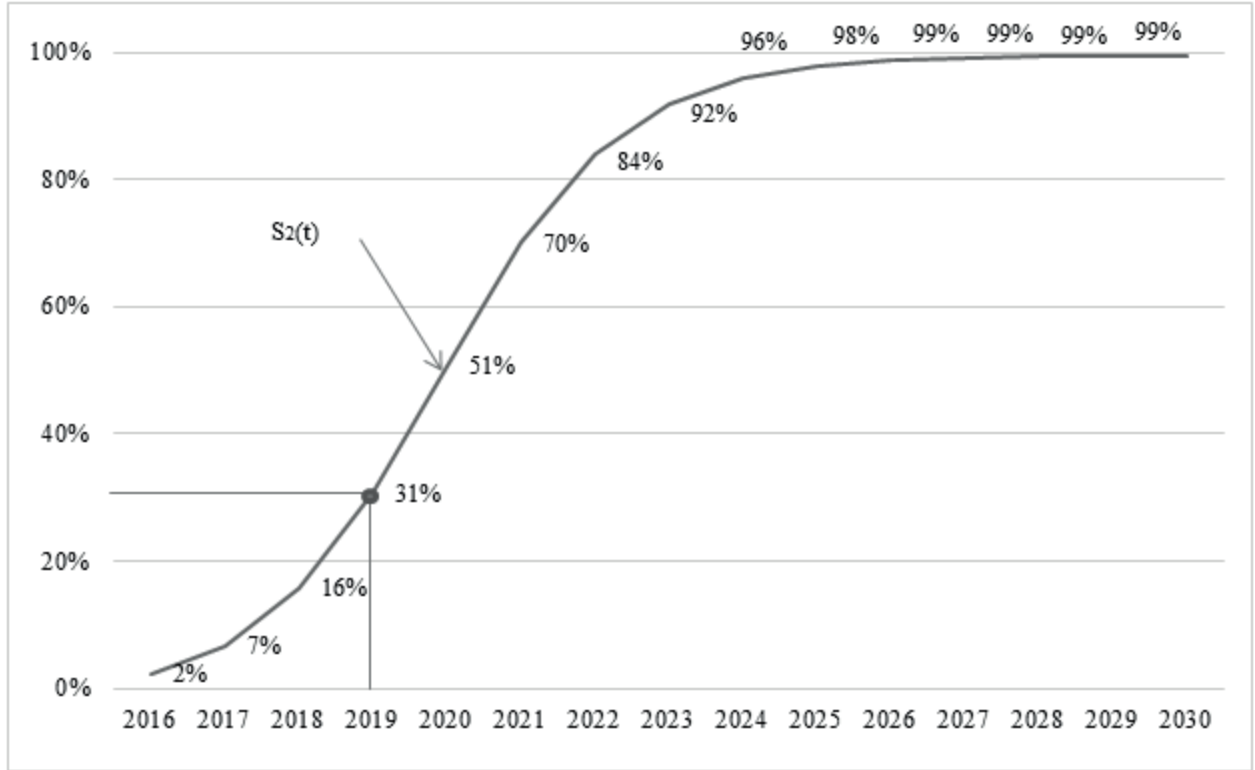

Figura 6. Curva de difusión esperada para la adopción del reloj inteligente en Brasil

Figure 6. Expected diffusion curve for the adoption of smart watch in Brazil

Fuente: elaboración propia.

Mientras que las proyecciones para países de primer mundo, como España y EE.UU. (ver Figuras 7 y 8) muestran que con un factor de innovación más fuerte, la velocidad con la que se difunde la adopción de un nuevo producto a través del tiempo es crítica en los primeros cuatro años entre el $40 \%$ para EE. UU. y $50 \%$ España, lo que representa una masa crítica potencial de consumidores de tipo innovador de 120 millones y 20 millones respectivamente.

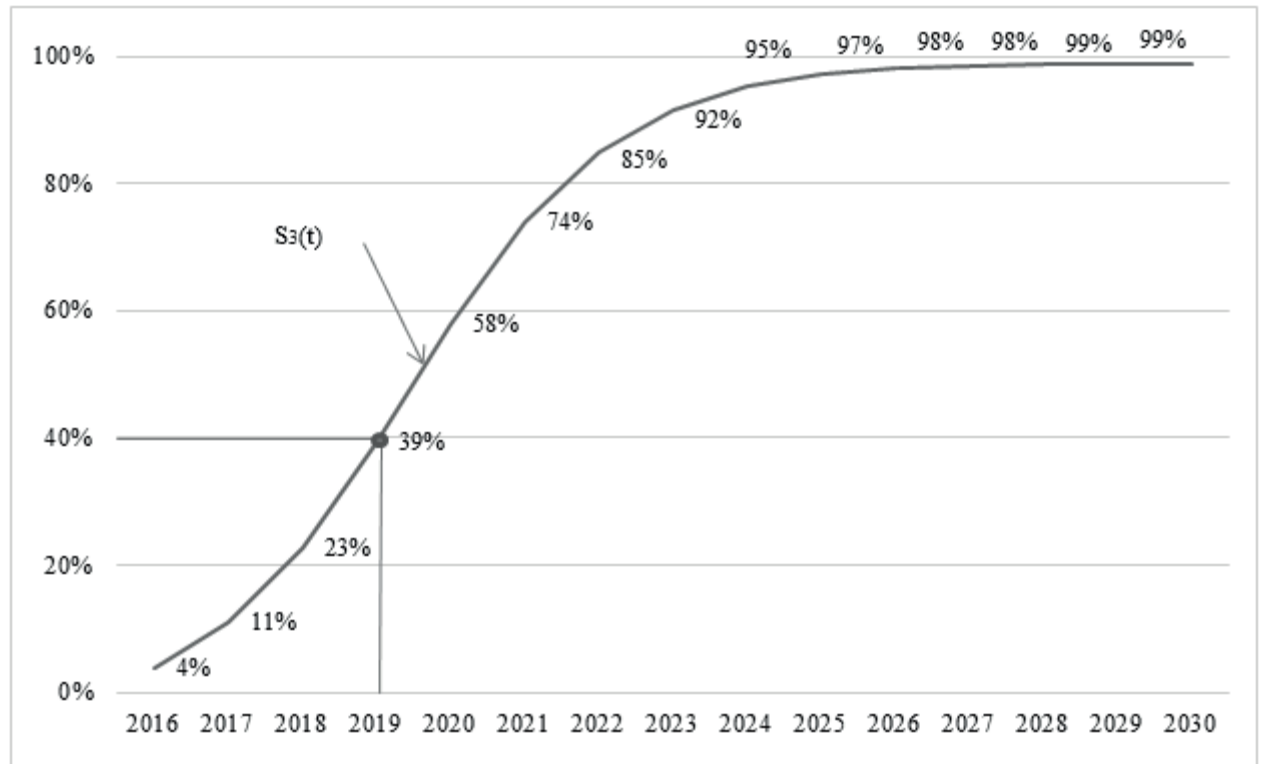

Figura 7. Curva de difusión esperada para la adopción del reloj inteligente en España

Figura 7. Expected diffusion curve for the adoption of smart watch in Spain

Fuente: elaboración propia. 


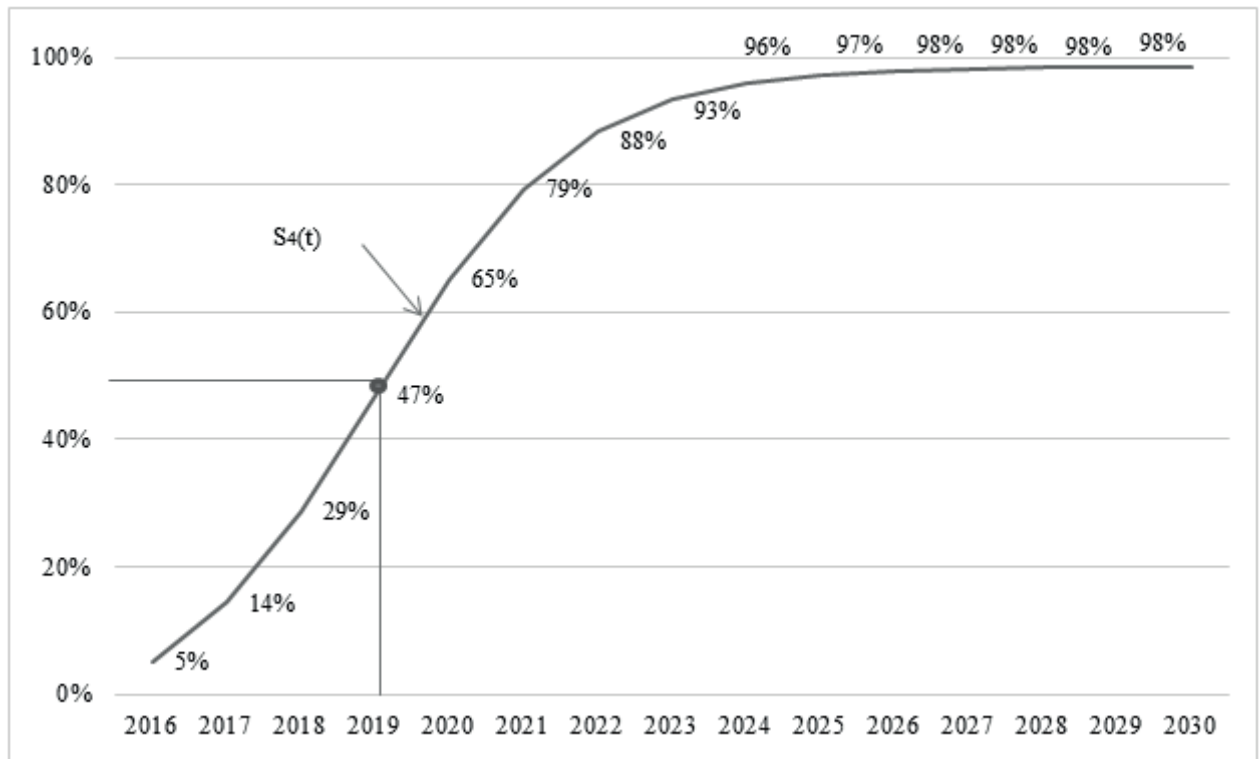

Figura 8. Curva de difusión esperada para la adopción del reloj inteligente en EE. UU

Figure 8. Expected diffusion curve for the adoption of smart watch in USA

Fuente: elaboración propia.

Se observa que en el periodo de los cuatro a los siete años, el porcentaje de masa crítica en los cuatro países de esta comparativa alcanza el $80 \%$, por lo que posterior el factor de imitación no representa una diferencia tan significativa en términos relativos.

\section{CONCLUSIONES}

En consecuencia, conocer el patrón de adopción de una innovación en un país no es automáticamente trasladable a otro país, especialmente si tienen un distinto nivel en desarrollo económico. Así también, debido a los coeficientes de innovación de mayor magnitud en países desarrollados como EE. UU. y España contra los países en desarrollo México y Brasil, en los primeros se alcanza más rápidamente el pico de ventas, lo que es una barrera a nuevos jugadores entrantes que deseen empezar con el arranque y comercialización de un nuevo producto introducido en el mercado, ya que los primeros se posicionan rapidamente al cabo de pocos años y con un porcentaje importante de la masa crítica que lo pueda adquirir.
Una extensión del modelo de Bass consiste en incluir el efecto de productos complementarios, es decir, que la compra de un producto es contingente a la compra de otro producto básico la compra de videojuegos (software), depende de la disponibilidad de consolas (hardware), con lo cual para porspectar el mercado de los primeros es necesario prospectar el mercado de los segundos, una comparativa similar se puede dar tanto entre el reloj como el telefono inteligente.

Un área fértil de investigación es la aplicación del modelo de Bass ampliado para analizar la influencia que los nuevos medios, como Internet e Internet de las cosas, pueden generar en la difusión de un nuevo producto, con el cual es probable se produzca un efecto favorable y no tanto para su difusión en mayor rapidez o subsecuente fracaso en menor tiempo.

Finalmente, el uso de la prospectiva independientemente del grado de confianza que aporte, seguirá siendo útil para la generación de políticas públicas y planeación estratégica de las empresas como parte de sus 
decisiones en inversión, en consecuencia, la constante necesidad de mejorar los métodos para realizarla.

\section{REFERENCIAS}

Baltes, H.; Brand, O.; Fedder, G.; Hierold, C.; Korvink, J. y Tabata, O. (2004). Enabling Technology for MEMS and Nanodevices. Weinheim: Wiley-VCH.

Bass, F. (2004). Comments on "A New Product Growth Model for Consumer Durables". Management Science, 1833-1840.

Bonato, P.; Sherril, D. M.; Mork, P. J. y Westgaard, R. H. (2003). Data Mining of Motor Patterns Recorder with Wearable Technology. IEEE Engineering in Medicine and Biology Magazine.

Bower, J. L. y Christensen, C. M. (1995). Disruptive Technologies: Catching the Wave. Harvard Business Review, 43-53.

Ehrfeld, W. y Ehrfeld, U. (2001). Progress and profit through micro technologies. Commercial applications of MEMS / MOEMS. Mainz: SPIE Vol. 4557.

Frank, R. (2000). Understanding smart sensors. Norwood: Artech House.

Fujita, H. (1996). Future of actuators and microsystems. Sensors and Actuators A: Physical, A56, pp. 105.111.
Fujita, H. (1998). Microactuators and micromachines. Proc. IEEE, 86(8), 721-732.

Kondratieff, N. (1984). The Long Wave Cycle. New York: E. P. Dutton.

Mahajan, V. y Muller, E. (1979). Innovation Difussion and New Product Growth Models in Marketing. Journal of Marketing, 43, 5568.

Malshe, A. P.; O’Neal, C.; Singh, S. B.; Brown, W. D.; Eaton, W. P. y Miller, W. M. (1999). Challenges in the packaging of MEMS. International Journal of Microcircuits and Electronic Packaging, 22(3), 233-241.

MEMS \& Sensors for Wearables Report (2014). Recuperado de http://www.technology.ihs.com

Naisbitt, J. y Aburdene, P. (1990). Megatrends 2000. New York: Wm Morrow Co.

Ofek, E. (2005). Forecasting the Adoption of a New Product. HBS Case Collection.

Radojicic, V. D. y Markovic, G. Z. (2009). New Technology Forecasting Using the Bass Model. IEEE, 215-227.

Rogers, E. (2003). The Diffusion of Innovations. New York: The Free Press.

Wearable Sensor Market Report (2014). Recuperado de IHS Newsroom: http://press.ihs.com/pressrelease/technology/wearable-sensormarket-expand-sevenfold-five-years 